Chaos 20 (2010) 023127

\title{
Fractional Dissipative Standard Map
}

\author{
Vasily E. Tarasov \\ Courant Institute of Mathematical Sciences, New York University, \\ 251 Mercer St., New York, NY 10012, USA \\ Skobeltsyn Institute of Nuclear Physics, \\ Moscow State University, Moscow 119991, Russia \\ M. Edelman \\ Courant Institute of Mathematical Sciences, New York University, \\ 251 Mercer St., New York, NY 10012, USA \\ Department of Physics, Stern College at Yeshiva University, \\ 245 Lexington Ave, New York, NY 10016, USA
}

\begin{abstract}
Using kicked differential equations of motion with derivatives of noninteger orders, we obtain generalizations of the dissipative standard map. The main property of these generalized maps, which are called fractional maps, is long-term memory. The memory effect in the fractional maps means that their present state of evolution depends on all past states with special forms of weights. Already a small deviation of the order of derivative from the integer value corresponding to the regular dissipative standard map (small memory effects) leads to the qualitatively new behavior of the corresponding attractors. The fractional dissipative standard maps are used to demonstrate a new type of fractional attractors in the wide range of the fractional orders of derivatives.
\end{abstract}


Discrete maps are widely used to study the general properties of dynamical systems. In those cases when they can be derived from differential equations, their analysis gives the exact properties of the corresponding systems. In this article we derive discrete maps (fractional maps) from the fractional differential equations, which correspond to the fractional generalizations of the dissipative standard map [1]. We demonstrate how the attractors of the fractional maps are different from the attractors of the dissipative standard map.

\section{INTRODUCTION}

There is a number of distinct areas of physics where basic problems can be reduced to the study of simple discrete maps. Discrete maps as substitutes of differential equations have been used to study evolution problems in Refs. [2-6]. They lead to a simpler formalism, which is particularly useful in simulations. The dissipative standard map is one of the most widely studied maps. In this paper we consider fractional generalizations of the dissipative standard map which are described by fractional differential equations [7-9].

The treatment of nonlinear dynamics in terms of discrete maps is a very important step in understanding the qualitative behavior of systems described by differential equations. The derivatives of noninteger orders are a natural generalization of the ordinary differentiation of the integer order. The fractional differentiation with respect to time is characterized by long-term memory effects which correspond to intrinsic dissipative processes in physical systems. The application of memory effects to discrete maps means that their present state of evolution depends on all past states [10 15, 17].

Discrete maps with memory can be derived (see Ref. [17]) from equations of motion with fractional derivatives. In Ref. [17] a fractional generalization of the standard map has been derived from a fractional differential equation. A fractional generalization of the dissipative standard map was also suggested in [17]. Unfortunately, in that generalization a dissipation was introduced by the change of the variable $p_{n} \rightarrow-b p_{n}$. The map equations were not directly connected with a fractional equation of motion. In this paper we propose two generalizations of the dissipative standard map. The first one is derived from a differential equation with fractional damped kicks. The second generalization of the dissipative standard map is derived from a fractional differential equation (kicks are not fractional) . A nonlinear 
system with fractional derivatives perturbed by a periodic force exhibits a new type of chaotic motion which can be called the fractional chaotic attractor [16]. Fractional discrete maps [17] are used to study new types of attractors of fractional dynamics described by kicked fractional equations. In this paper some fractional differential equations of motion of kicked systems with friction are considered. Corresponding discrete maps with memory are derived from these equations. The fractional generalizations of the dissipative standard map are suggested and these maps are used in computer simulations.

\section{DISCRETE MAPS WITHOUT MEMORY}

In this section, a brief review of discrete maps is considered to fix notations and provide convenient references. For details, see Refs. [2 $[6]$.

\section{A. Standard map}

Let us consider the equation of motion

$$
\ddot{x}+K \sin (x) \sum_{n=0}^{\infty} \delta(t-n)=0,
$$

in which perturbation is a periodic sequence of delta-function-type pulses (kicks) following with period $T=1, K$ is the amplitude of the pulses. This equation can be presented in the Hamiltonian form

$$
\dot{x}=p, \quad \dot{p}+K \sin (x) \sum_{n=0}^{\infty} \delta(t-n)=0 .
$$

It is well-known that these equations can be represented (see, for example, Chapter 5 in Ref. [3]) in the form of discrete map

$$
\begin{gathered}
x_{n+1}=x_{n}+p_{n+1}, \\
p_{n+1}=p_{n}-K \sin \left(x_{n}\right) .
\end{gathered}
$$

Equations (3) and (4) are called the standard map. This map is also called the Chirikov $\operatorname{map}[4]$. 


\section{B. Dissipative standard map}

The dissipative standard map [1, 18, 19] is

$$
\begin{gathered}
X_{n+1}=X_{n}+\mu Y_{n+1}+\Omega, \\
Y_{n+1}=e^{-q}\left[Y_{n}+\varepsilon \sin \left(X_{n}\right)\right],
\end{gathered}
$$

where $\mu=\left(e^{q}-1\right) / q$. The dissipative standard map is also called the Zaslavsky map. Note that a shift $\Omega$ does not play an important role and it can be put to zero $(\Omega=0)$. The dissipative standard map with $\Omega=0$ can be represented by the equations

$$
\begin{gathered}
X_{n+1}=X_{n}+P_{n+1}, \\
P_{n+1}=-b P_{n}-Z \sin \left(X_{n}\right) .
\end{gathered}
$$

For the parameters

$$
Z=-\varepsilon \mu e^{-q}, \quad P_{n}=\mu Y_{n}, \quad b=-e^{-q}
$$

equations (7) and (8) give Eqs. (5) and (6) with $\Omega=0$.

For $b=-1$ and $Z=K$, we get the standard map which is described by Eqs. (3) and (4) with $T=1$.

Note that for large $q \rightarrow \infty$ (for small $b \rightarrow 0$ ) Eqs. (7) and (8) with $Z=-K$ shrink to the one-dimensional sine map proposed by Arnold [20],

$$
X_{n+1}=X_{n}+K \sin \left(X_{n}\right)
$$

\section{Kicked damped rotator map}

The equation of motion for a kicked damped rotator is

$$
\ddot{x}+q \dot{x}=K G(x) \sum_{n=0}^{\infty} \delta(t-n T) .
$$

It is well known [5] that Eq. (11) gives the two-dimensional map

$$
\begin{gathered}
x_{n+1}=x_{n}+\frac{1-e^{-q T}}{q}\left[p_{n}+K G\left(x_{n}\right)\right], \\
p_{n+1}=e^{-q T}\left[p_{n}+K G\left(x_{n}\right)\right] .
\end{gathered}
$$


This map is known as the kicked damped rotator map. The phase volume shrinks each time step by a factor $\exp (-q)$. The map is defined by two important parameters, dissipation constant $q$ and force amplitude $K$. These equations can be rewritten in the form

$$
\begin{gathered}
x_{n+1}=x_{n}+\frac{e^{q T}-1}{q} p_{n+1}, \\
p_{n+1}=e^{-q T}\left[p_{n}+K G\left(x_{n}\right)\right] .
\end{gathered}
$$

It is easy to see that these equations give the dissipative standard map (7) and (8) with $\Omega=0$ if we use

$$
X_{n}=x_{n}, \quad Y_{n}=p_{n}, \quad \varepsilon=K, \quad T=1, \quad G(x)=\sin (x) .
$$

This allows us to derive dissipative standard map (5) and (6) from the differential equation,

$$
\ddot{X}+q \dot{X}=\varepsilon \sin (X) \sum_{n=0}^{\infty} \delta(t-n) .
$$

These equations give the discrete map defined by Eqs. (5) and (6) with $\Omega=0$.

\section{FRACTIONAL STANDARD MAP AND DISSIPATION}

A fractional generalization of the differential equation (11) has been suggested in Ref.

[17]. The discrete map which corresponds to the fractional equation of order $1<\alpha \leq 2$ was derived. This map can be considered as a generalization of the standard map for the case $1<\alpha \leq 2$.

We consider a fractional generalization of Eq. (11) in the form

$$
{ }_{0} D_{t}^{\alpha} x+K \sin (x) \sum_{n=0}^{\infty} \delta(t-n)=0, \quad(1<\alpha \leq 2),
$$

where ${ }_{0} D_{t}^{\alpha}$ is the Riemann-Liouville fractional derivative [7-9], which is defined by

$$
{ }_{0} D_{t}^{\alpha} x=D_{t}^{2}{ }_{0} I_{t}^{2-\alpha} x=\frac{1}{\Gamma(2-\alpha)} \frac{d^{2}}{d t^{2}} \int_{0}^{t} \frac{x(\tau) d \tau}{(t-\tau)^{\alpha-1}}, \quad(1<\alpha \leq 2) .
$$

Here we use the notation $D_{t}^{2}=d^{2} / d t^{2}$, and ${ }_{0} I_{t}^{\alpha}$ is a fractional integration [7 9$]$.

Defining the momentum as

$$
p(t)={ }_{0} D_{t}^{\alpha-1} x(t)
$$


and using the initial conditions

$$
\left({ }_{0} D_{t}^{\alpha-1} x\right)(0+)=p_{1}, \quad\left({ }_{0} D_{t}^{\alpha-2} x\right)(0+)=b,
$$

it is possible to derive the equation for the fractional standard map.

Proposition 1. The fractional differential equation of the kicked system (15) is equivalent to the discrete map

$$
\begin{gathered}
x_{n+1}=\frac{1}{\Gamma(\alpha)} \sum_{k=0}^{n} p_{k+1} V_{\alpha}(n-k+1)+\frac{b}{\Gamma(\alpha-1)}(n+1)^{\alpha-2}, \\
p_{n+1}=p_{n}-K \sin \left(x_{n}\right), \quad(1<\alpha \leq 2),
\end{gathered}
$$

where the function $V_{\alpha}(z)$ is defined by

$$
V_{\alpha}(z)=z^{\alpha-1}-(z-1)^{\alpha-1}
$$

Proof of this Proposition is given in Ref. [21].

A fractional generalization of the dissipative standard map suggested in Refs. [1, 18] can be defined by

$$
\begin{gathered}
x_{n+1}=\frac{1}{\Gamma(\alpha)} \sum_{k=0}^{n} p_{k+1} V_{\alpha}(n-k+1), \quad(1<\alpha \leq 2), \\
p_{n+1}=-b p_{n}-Z \sin \left(x_{n}\right),
\end{gathered}
$$

where the parameters are defined by conditions (91). For $b=-1$ and $Z=K$ Eqs. (21) and (22) give the fractional standard map with $T=1$. Note that this fractional dissipative standard map is not derived from a fractional differential equation. This map is derived by $p_{n} \rightarrow-b p_{n}$ in the fractional standard map. Fractional dissipative standard map can be derived from fractional differential equations. In this paper, we derive two fractional generalizations of the dissipative standard map which are obtained from fractional differential equations.

\section{FRACTIONAL DERIVATIVE IN THE KICKED TERM AND THE FIRST FRACTIONAL DISSIPATIVE STANDARD MAP}

In this section we suggest the first fractional generalization of differential equation (11) for a kicked damped rotator. In this generalization we introduce a fractional derivative in 
the kicked damped term, i.e. the term of a periodic sequence of delta-function-type pulses (kicks), and derive the corresponding discrete map.

Consider the fractional generalization of equation (111) in the form

$$
D_{t}^{2} X(t)-q D_{t}^{1} X(t)=\varepsilon \sin \left({ }_{0}^{C} D_{t}^{\beta} X\right) \sum_{n=0}^{\infty} \delta(t-n), \quad(0 \leq \beta<1),
$$

where $q \in \mathbb{R}$, and ${ }_{0}^{C} D_{t}^{\beta}$ is the Caputo fractional derivative [9] of the order $0 \leq \beta<1$ defined by

$$
{ }_{0}^{C} D_{t}^{\beta} X={ }_{0} I_{t}^{1-\beta} D_{t}^{1} X=\frac{1}{\Gamma(1-\beta)} \int_{0}^{t} \frac{d \tau}{(t-\tau)^{\beta}} \frac{d X(\tau)}{d \tau}, \quad(0 \leq \beta<1) .
$$

Here we use the notation $D_{t}^{1}=d / d t$, and ${ }_{0} I_{t}^{\alpha}$ is a fractional integration [7 9 ]. For $\beta=0$ fractional equation (23) gives equation (11). Note that we use the minus on the left-hand side of Eq. (23), where $q$ can be a positive or negative value. Fractional derivative ${ }_{0}^{C} D_{t}^{\beta} X$ is presented in the kicked damped term.

Proposition 2. The fractional differential equation of the kicked system (23) is equivalent to the discrete map

$$
\begin{gathered}
X_{n+1}=X_{n}+\frac{1-e^{-q}}{q} Y_{n+1}, \\
Y_{n+1}=e^{q}\left[Y_{n}+\varepsilon \sin \left(\frac{1}{\Gamma(1-\beta)} \sum_{k=0}^{n-1} Y_{k+1} W_{2-\beta}(q, k-n)\right)\right],
\end{gathered}
$$

where the functions $W_{2-\beta}(a, b)$ are defined by

$$
W_{2-\beta}(a, b)=a^{\beta-1} e^{a(b+1)}[\Gamma(1-\beta, a b)-\Gamma(1-\beta, a(b+1))]
$$

and $\Gamma(a, b)$ is the incomplete Gamma function

$$
\Gamma(a, b)=\int_{b}^{\infty} y^{a-1} e^{-y} d y
$$

Proof. Fractional equation (23) can be presented in the Hamiltonian form

$$
\begin{gathered}
\dot{X}=Y, \\
\dot{Y}-q Y=\varepsilon \sin \left({ }_{0}^{C} D_{t}^{\beta} X\right) \sum_{n=0}^{\infty} \delta(t-n),
\end{gathered}
$$

where $0<\beta<0$, and $q \in \mathbb{R}$.

Between any two kicks,

$$
\dot{Y}-q Y=0
$$


For $t \in\left(t_{n}+0, t_{n+1}-0\right)$, the solution of Eq. (29) is

$$
Y\left(t_{n+1}-0\right)=Y\left(t_{n}+0\right) e^{q}
$$

Let us use the notations $t_{n}=n T$, with $T=1$ and

$$
\begin{gathered}
X_{n}=X\left(t_{n}-0\right)=\lim _{\epsilon \rightarrow 0} X(n-\epsilon), \\
Y_{n}=Y\left(t_{n}-0\right)=\lim _{\epsilon \rightarrow 0} Y(n-\epsilon) .
\end{gathered}
$$

For $t \in\left(t_{n}-\epsilon, t_{n+1}-\epsilon\right)$, the general solution of Eq. (28) is

$$
Y(t)=Y_{n} e^{q\left(t-t_{n}\right)}+\varepsilon \sum_{m=0}^{\infty} \sin \left({ }_{0}^{C} D_{t_{m}}^{\beta} X\right) \int_{t_{n}-\epsilon}^{t} d \tau e^{q(t-\tau)} \delta(\tau-m)
$$

Then

$$
Y_{n+1}=e^{q}\left[Y_{n}+\varepsilon \sin \left({ }_{0}^{C} D_{t_{n}}^{\beta} X\right)\right] .
$$

Using Eq. (33), the integration of the first equation of (28) gives

$$
X_{n+1}=X_{n}-\frac{1-e^{q}}{q}\left[Y_{n}+\varepsilon \sin \left({ }_{0}^{C} D_{t_{n}}^{\beta} X\right)\right]
$$

Let us consider the Caputo fractional derivative from Eqs. (33) and (34). It is defined by the equation

$$
{ }_{0}^{C} D_{t_{n}}^{\beta} X={ }_{0} I_{t}^{1-\beta} D_{t}^{1} X=\frac{1}{\Gamma(1-\beta)} \int_{0}^{t_{n}} \frac{d \tau}{\left(t_{n}-\tau\right)^{\beta}} \frac{d X(\tau)}{d \tau}, \quad(0 \leq \beta<1) .
$$

Using $Y(\tau)=d X(\tau) / d \tau$, this relation can be rewritten as

$$
{ }_{0}^{C} D_{t_{n}}^{\beta} X=\frac{1}{\Gamma(1-\beta)} \sum_{k=0}^{n-1} \int_{t_{k}}^{t_{k+1}} \frac{Y(\tau) d \tau}{\left(t_{n}-\tau\right)^{\beta}},
$$

where $t_{k+1}=t_{k}+1=(k+1)$, and $t_{k}=k$, such that $t_{0}=0$. For $\tau \in\left(t_{k}, t_{k+1}\right)$, equations (30) and (31) give

$$
\begin{gathered}
Y(\tau)=Y\left(t_{k}+0\right) e^{q\left(\tau-t_{k}\right)}=Y\left(t_{k+1}-0\right) e^{-q} e^{q\left(\tau-t_{k}\right)}= \\
=Y_{k+1} e^{q\left(\tau-t_{k}-1\right)}=Y_{k+1} e^{q\left(\tau-t_{k+1}\right)}
\end{gathered}
$$

Then

$$
\int_{t_{k}}^{t_{k+1}} \frac{Y(\tau) d \tau}{\left(t_{n}-\tau\right)^{\beta}}=Y_{k+1} \int_{t_{k}}^{t_{k+1}} e^{q\left(\tau-t_{k+1}\right)}\left(t_{n}-\tau\right)^{-\beta} d \tau=
$$




$$
\begin{gathered}
=Y_{k+1} \int_{t_{n}-t_{k+1}}^{t_{n}-t_{k}} e^{q\left(t_{n}-t_{k+1}-z\right)} z^{-\beta} d z=Y_{k+1} e^{q\left(t_{n}-t_{k+1}\right)} \int_{t_{n}-t_{k+1}}^{t_{n}-t_{k}} z^{-\beta} e^{-q z} d z= \\
=Y_{k+1} q^{\beta-1} e^{q(n-k-1)} \int_{q\left(t_{n}-t_{k+1}\right)}^{q\left(t_{n}-t_{k}\right)} y^{-\beta} e^{-y} d y .
\end{gathered}
$$

As a result, equation (36) gives

$$
\begin{gathered}
\int_{t_{k}}^{t_{k+1}} \frac{Y(\tau) d \tau}{\left(t_{n}-\tau\right)^{\beta}}= \\
=Y_{k+1} q^{\beta-1} e^{q(n-k-1)}\left[\Gamma\left(1-\beta, q\left(t_{n}-t_{k+1}\right)\right)-\Gamma\left(1-\beta, q\left(t_{n}-t_{k}\right)\right)\right] .
\end{gathered}
$$

Here $\Gamma(a, b)$ is the incomplete Gamma function (27), where $a$ and $b$ are complex numbers. Using (35) and (37), we obtain

$$
{ }_{0}^{C} D_{t_{n}}^{\beta} X=\frac{1}{\Gamma(1-\beta)} \sum_{k=0}^{n-1} Y_{k+1} W_{2-\beta}(q, k-n), \quad(0 \leq \beta<1),
$$

where

$$
W_{2-\beta}(a, b)=a^{\beta-1} e^{a(b+1)}[\Gamma(1-\beta, a b)-\Gamma(1-\beta, a(b+1))] .
$$

Substitution of Eq. (38) into Eqs. (33) and (34) gives

$$
\begin{gathered}
Y_{n+1}=e^{q}\left[Y_{n}+\varepsilon \sin \left(\frac{1}{\Gamma(1-\beta)} \sum_{k=0}^{n-1} Y_{k+1} W_{2-\beta}(q, k-n)\right)\right], \\
X_{n+1}=X_{n}-\frac{1-e^{q}}{q}\left[Y_{n}+\varepsilon \sin \left(\frac{1}{\Gamma(1-\beta)} \sum_{k=0}^{n-1} Y_{k+1} W_{2-\beta}(q, k-n)\right)\right] .
\end{gathered}
$$

Equations (40) and (41) can be presented in the form of Eqs. (25) and (26).

This ends the proof.

The iteration equations (25) and (26) define a fractional generalization of the dissipative standard map. For $\beta=0$ this map gives the Zaslavsky map (5) and (6) with

$$
\mu=\left(1-e^{-q}\right) / q
$$

and $\Omega=0$. 


\section{FRACTIONAL DERIVATIVE IN THE UNKICKED TERMS AND THE SEC- OND FRACTIONAL DISSIPATIVE STANDARD MAP}

In this section we suggest a fractional generalization of the differential equation for a kicked damped rotator with fractional derivatives in the unkicked terms and derive the corresponding discrete map.

We consider the fractional generalization of equation (11) in the form

$$
{ }_{0} D_{t}^{\alpha} X(t)-q_{0} D_{t}^{\beta} X(t)=\varepsilon \sin (X) \sum_{n=0}^{\infty} \delta(t-n),
$$

where

$$
q \in \mathbb{R}, \quad 1<\alpha \leq 2, \quad \beta=\alpha-1
$$

and ${ }_{0} D_{t}^{\alpha}$ is the Riemann-Liouville fractional derivative [7 -9], which is defined by Eq. (16). This equation has fractional derivatives in the unkicked terms, i.e. on the left-hand side of Eq. (43). We use the minus in the left-hand side of Eq. (43), where $q$ can have a positive or negative value.

Proposition 3. The fractional differential equation of the kicked system (43) is equivalent to the discrete map

$$
\begin{gathered}
X_{n+1}=\frac{1}{\Gamma(\alpha-1)} \sum_{k=0}^{n} Y_{k+1} W_{\alpha}(q, k-n-1), \\
Y_{n+1}=e^{q}\left[Y_{n}+\varepsilon \sin \left(X_{n}\right)\right]
\end{gathered}
$$

where the functions $W_{\alpha}(a, b)$ are defined by

$$
W_{\alpha}(a, b)=a^{1-\alpha} e^{a(b+1)}[\Gamma(\alpha-1, a b)-\Gamma(\alpha-1, a(b+1))],
$$

and $\Gamma(a, b)$ is the incomplete Gamma function (27).

Proof. Let us define an auxiliary variable $\xi(t)$ such that

$$
{ }_{0}^{C} D_{t}^{2-\alpha} \xi=X(t)
$$

where ${ }_{0}^{C} D_{t}^{2-\alpha}$ is the Caputo fractional derivative (24). Using

$$
{ }_{0} I_{t}^{2-\alpha}{ }_{0}^{C} D_{t}^{2-\alpha} \xi=\xi(t)-\xi(0), \quad(0 \leq 2-\alpha<1),
$$


we obtain

$$
{ }_{0} D_{t}^{\alpha} X=D_{t}^{2}{ }_{0} I_{t}^{2-\alpha} X=D_{t}^{2}{ }_{0} I_{t}^{2-\alpha}{ }_{0}^{C} D_{t}^{2-\alpha} \xi=D_{t}^{2}(\xi(t)-\xi(0))=D_{t}^{2} \xi,
$$

and

$$
\begin{gathered}
{ }_{0} D_{t}^{\beta} X=D_{t}^{1}{ }_{0} I_{t}^{1-\beta} X=D_{t}^{1}{ }_{0} I_{t}^{2-\alpha} X= \\
=D_{t}^{1}{ }_{0} I_{t}^{2-\alpha}{ }_{0}^{C} D_{t}^{2-\alpha} \xi=D_{t}^{1}(\xi(t)-\xi(0))=D_{t}^{1} \xi .
\end{gathered}
$$

Substitution of Eqs. (49), (50), and (47) into Eq. (43) gives

$$
D_{t}^{2} \xi-q D_{t}^{1} \xi=\varepsilon \sin \left({ }_{0}^{C} D_{t}^{2-\alpha} \xi\right) \sum_{n=0}^{\infty} \delta(t-n), \quad(1<\alpha \leq 2) .
$$

The fractional equation (51) can be presented in the Hamiltonian form

$$
\begin{gathered}
\dot{\xi}=Y, \\
\dot{Y}-q Y=\varepsilon \sin \left({ }_{0}^{C} D_{t}^{2-\alpha} \xi\right) \sum_{n=0}^{\infty} \delta(t-n), \quad(1<\alpha<2, \quad q \in \mathbb{R}) .
\end{gathered}
$$

Using Eq. (26) of Proposition 2, we obtain

$$
Y_{n+1}=e^{q}\left[Y_{n}+\varepsilon \sin \left(\frac{1}{\Gamma(\alpha-1)} \sum_{k=0}^{n-1} Y_{k+1} W_{\alpha}(q, k-n)\right)\right] .
$$

For $\left(X_{n}, Y_{n}\right)$, we use equation (38) in the form

$$
X_{n}={ }_{0}^{C} D_{t_{n}}^{2-\alpha} \xi=\frac{1}{\Gamma(\alpha-1)} \sum_{k=0}^{n-1} Y_{k+1} W_{\alpha}(q, k-n) .
$$

As a result, we have

$$
\begin{gathered}
X_{n+1}=\frac{1}{\Gamma(\alpha-1)} \sum_{k=0}^{n} Y_{k+1} W_{\alpha}(q, k-n-1), \\
Y_{n+1}=e^{q}\left[Y_{n}+\varepsilon \sin \left(X_{n}\right)\right]
\end{gathered}
$$

where $W_{\alpha}(a, b)$ is defined in Eq. (46). This ends the proof.

If we use the variables

$$
P_{n}=\mu Y_{n}, \quad b=-e^{q}, \quad Z=-\mu \varepsilon e^{q},
$$



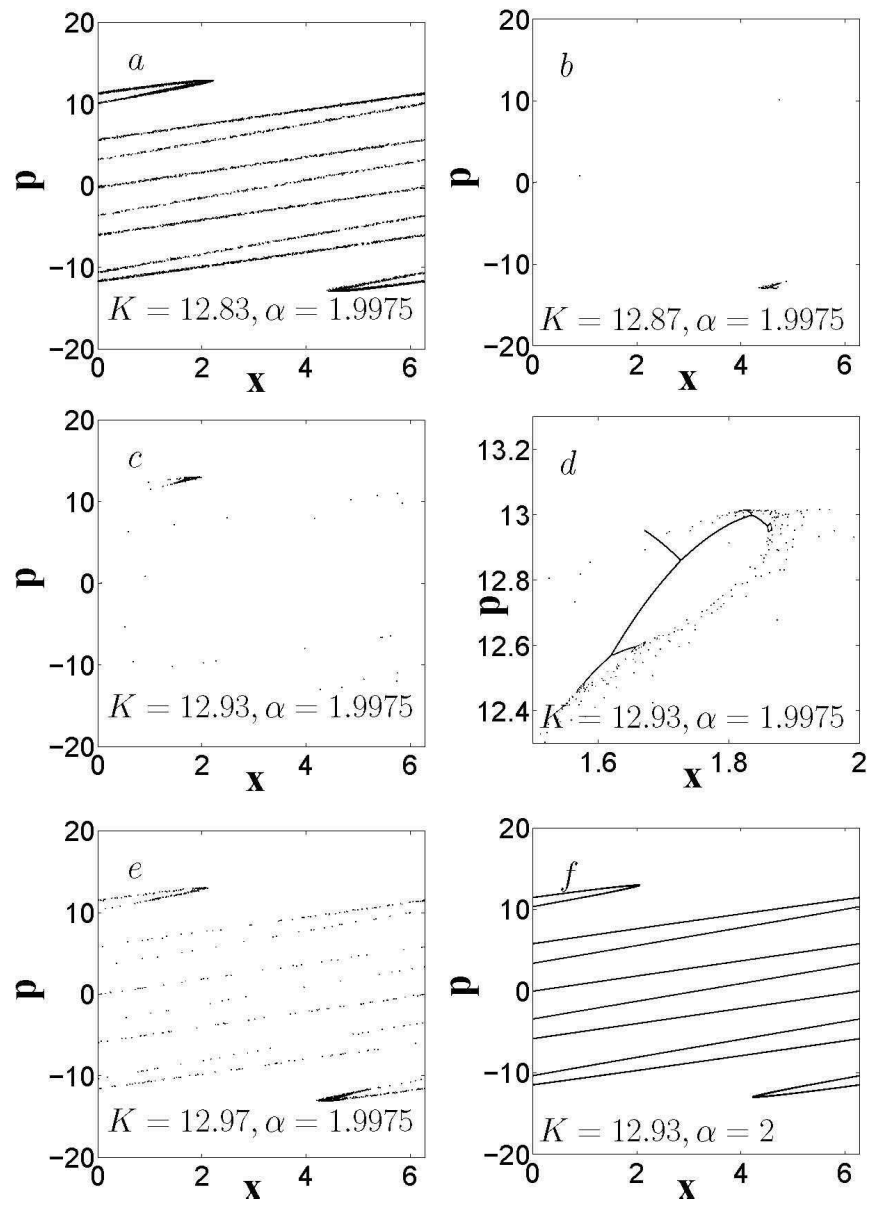

FIG. 1: Structures of the chaotic attractors for different values of $K$ obtained after $10^{5}$ iterations $(\Gamma=5, \Omega=0) ; \alpha=1.9975$ in (a)-(e). (a) $K=12.83$; (b) $K=12.87$; (c) $K=12.93$; (d) zoom of (c); (e) $K=12.97$; (f) $\alpha=2, K=12.93$.

then equations (44) and (45) give

$$
\begin{gathered}
X_{n+1}=\frac{\mu^{-1}}{\Gamma(\alpha-1)} \sum_{k=0}^{n} P_{k+1} W_{\alpha}(q, k-n-1) . \\
P_{n+1}=-b P_{n}-Z \sin \left(X_{n}\right),
\end{gathered}
$$

These equations can be considered as a fractional generalization of the dissipative standard map equations (7) and (8) with $\Omega=0$. For $\alpha=2$, this fractional dissipative standard map 

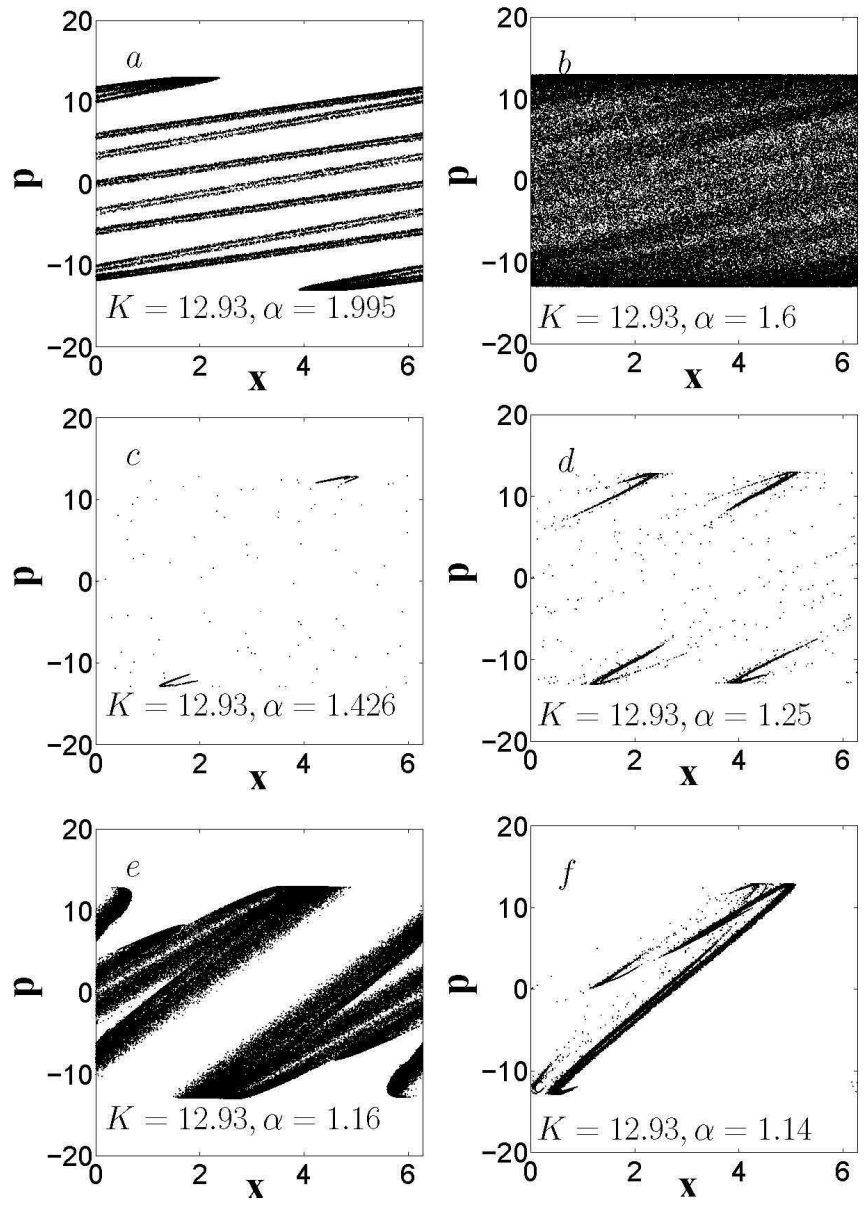

FIG. 2: Structures of the fractional chaotic attractors for $K=12.93$ and different values of $\alpha$ obtained after $10^{5}$ iterations $(\Gamma=5, \Omega=0)$.

gives the dissipative standard map that is described by Eqs. (7) and (8)).

\section{NUMERICAL SIMULATIONS}

Numerical simulations were performed for the second fractional dissipative standard map (Eqs. (55) and (56)). First we used our code to reproduce the results presented in Fig. 1 from [19] for the structures of the chaotic attractors of the dissipative standard map at the 
window of the ballistic motion near $K \approx 4 \pi(q=-5, K=\varepsilon \exp (q)$, and used in Ref. [19] $\Gamma$ is equal to $-q$ ) for the fractional standard map with $\alpha=2$ and obtained a perfect agreement (an example is given in Fig. 1(f)). As $\alpha$ decreases slightly from $\alpha=2$ to $\alpha=1.9975$, the window of the ballistic motion shrinks and moves to the higher values of $K$. Already for $\alpha=1.9975$ in Figs. 1(a)-1(e) the ballistic motion appears for $K>12.86$ and disappears at $K=12.97$. The window is completely closed at $\alpha \approx 1.9969$. The structures of two symmetric attractors with disjoint basins which appear within the window (Figs. 1(b) and $1(c))$ is also very different from the structures of the dying attractors of the dissipative standard map [1, 19]. The attractor in Fig. 1(d) evolves from period 8 trajectory to period 4, period 2, and, finally, period 1 trajectory slowly moving in the direction of the upper left corner with the step of the order of $10^{-7}$.

When $\alpha$ decreases further, the structures of the fractional chaotic attractors evolve in the manner presented in Fig. 2, where one can find one-scroll, two-scroll, and four-scroll fractional chaotic attractors, strongly deviating from the chaotic attractor of the dissipative standard map Fig. 1(f) (see also [19]). The problem of existence of multiscroll fractional chaotic attractors was considered in Ref. [22] but for the fractional differential equations with the Caputo derivatives. For values of $\alpha$ near 1 fractional chaotic attractor turns into period 2 and for smaller values period 1 attracting trajectories, Fig. 3.

\section{CONCLUSION}

The suggested discrete maps with memory are generalizations of the dissipative standard map. These maps are potentially useful for description of fractional dynamics of complex physical systems. We consider viscoelastic and dielectric materials as examples of complex media (physical systems) whose dynamics could be described by the fractional dissipative standard maps and corresponding fractional differential equations. This assumption is based on the following.

(1) It is well known that viscoelastic materials can be described by fractional differential equations (see, for example, Ref. [23]). The fractional dissipative standard map could be employed to model the one-dimensional simplification of the equations of viscoelastic materials in which a perturbation is a periodic sequence of delta-function-type pulses (kicks) following with some period. 


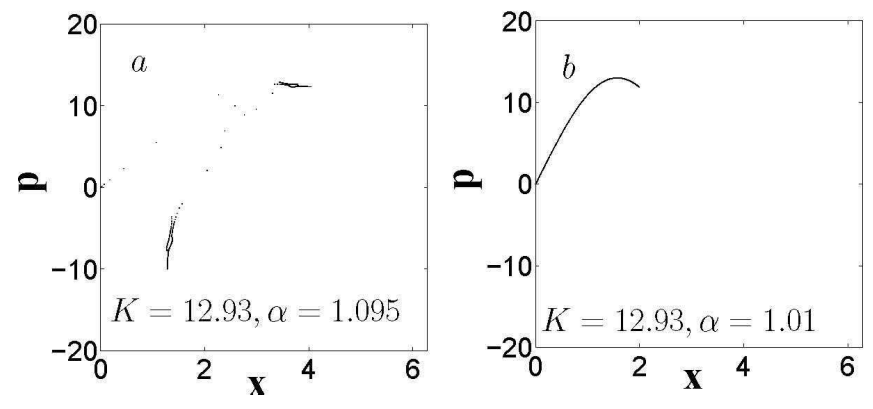

FIG. 3: Attracting trajectories for $K=12.93$ and small values of $\alpha$ obtained after $10^{5}$ iterations $(\Gamma=5, \Omega=0)$.

(2) For a wide class of dielectric materials the dielectric susceptibility follows a fractional power-law frequency dependence, which is called the "universal" response [24, 25] over extended frequency ranges. The electromagnetic fields in such dielectric media are described by differential equations with fractional time derivatives [26, 27]. These fractional equations for electromagnetic waves in dielectric media are common to a wide class of materials, regardless of the type of physical structure, or of the nature of the polarizing species. We assume that the fractional maps could be applied to dielectric media in which a perturbation 
is a periodic sequence of kicks.

The suggested fractional dissipative standard maps demonstrate a chaotic behavior with a new type of attractors. The interesting property of these fractional maps is long-term memory. As a result, a present state of evolution depends on all past states with the weight functions. The fractional dissipative standard maps are equivalent to the correspondent fractional kicked differential equations. Note that to derive discrete maps an approximation for fractional derivatives of these equations is not used.

Computer simulations of the suggested discrete maps with memory prove that the nonlinear dynamical systems, which are described by the equations with fractional derivatives, exhibit a new type of chaotic motion. This type of motion demonstrates a fractional generalization of attractors.

It has been shown in Ref. [28] that in the case $q=0$ (fractional standard map) the fractional discrete map demonstrates a new type of attractors such as slow converging and slow diverging trajectories, ballistic trajectories, fractal-like structures, and chaotic trajectories. At least one type of fractal-like sticky attractors in the chaotic sea can be observed [28] for the fractional standard map. The properties of stability and existence of the fractional

attractors in the asymptotic sense for $q=0$ have also been described in Ref. [28]. The attractors presented in Figs. 1-3 are quite different from the corresponding regular chaotic attractors and $q=0$ attractors. The detail classification of these attractors and corresponding chaotic motion will be considered in the nearest future and published in the next paper.

\section{Acknowledgments}

We express our gratitude to H. Weitzner for many comments and helpful discussions. This work was supported by the Office of Naval Research, Grant No. N00014-02-1-0056.

[1] G.M. Zaslavsky, Phys. Lett. A 69, 145 (1978); G.M. Zaslavsky and Kh.-R. Ya. Rachko, Sov. Phys. JETP 49, 1039 (1979).

[2] R.Z. Sagdeev, D.A. Usikov, G.M. Zaslavsky, Nonlinear Physics: From the Pendulum to Turbulence and Chaos (Harwood Academic Publishers, New-York, 1988). 
[3] G.M. Zaslavsky, Hamiltonian Chaos and Fractional Dynamics (Oxford University Press, Oxford, 2005).

[4] B.V. Chirikov, Phys. Rep. 52 (1979) 263-379.

[5] H.G. Schuster, Deterministic Chaos. An Introduction 2nd edn. (Physik Verlag, Weinheim, 1988).

[6] P. Collet, J.P. Eckman, Iterated Maps on the Interval as Dynamical System (Birkhauser, Basel, 1980).

[7] S.G. Samko, A.A. Kilbas, O.I. Marichev, Fractional Integrals and Derivatives Theory and Applications (Gordon and Breach, New York, 1993).

[8] I. Podlubny, Fractional Differential Equations (Academic Press, San Diego, 1999).

[9] A.A. Kilbas, H.M. Srivastava, J.J. Trujillo, Theory and Application of Fractional Differential Equations (Elsevier, Amsterdam, 2006).

[10] A. Fulinski, A.S. Kleczkowski, Physica Scripta 35 (1987) 119-122.

[11] E. Fick, M. Fick, G. Hausmann, Phys. Rev. A 44 (1991) 2469-2473.

[12] M. Giona, Nonlinearity 4 (1991) 991-925.

[13] K. Hartwich, E. Fick, Phys. Lett. A 177 (1993) 305-310.

[14] J.A.C. Gallas, Physica A 195 (1993) 417-430; "Erratum" Physica A 198 (1993) 339-339.

[15] A.A. Stanislavsky, Chaos 16 (2006) 043105.

[16] G.M. Zaslavsky, A.A. Stanislavsky, M. Edelman, Chaos $16 \quad$ (2006) 013102. (arXiv:nlin/0508018)

[17] V.E. Tarasov, G.M. Zaslavsky, J. Phys. A 41 (2008) 435101. (arXiv:1107.3953)

[18] G. Schmidt, B.W. Wang, Phys. Rev. A 32 (1985) 2994-2999.

[19] G.M. Zaslavsky,M. Edelman, Chaos 18 (2008) 033116. (arXiv:0805.1952).

[20] V. Arnold, Izv. Akad. Nauk. SSSR Ser. Mat. 25 (1961) 21-86 (in Russian); Amer. Math. Soc. Transl. (2) 46 (1965) 213-284.

[21] V.E. Tarasov Journal of Physics A 42 (2009) 465102. (arXiv:1107.4205)

[22] M.S. Tavazoei, M. Haeri, Physica D 237 (2008) 2628-2637.

[23] F. Mainardi, Fractional Calculus and Waves in Linear Viscoelasticity: An Introduction to Mathematical Models, (World Scientific Publishing, Singapore, 2010).

[24] A.K. Jonscher, Universal Relaxation Law, (Chelsea Dielectrics Press, London, 1996).

[25] A.K. Jonscher, J. Physics D Appl. Phys. 32 (1999) R57-R70. 
[26] V.E. Tarasov, Journal of Physics: Condensed Matter. 20 (2008) 175223. (arXiv:0907.2163)

[27] V.E. Tarasov, Journal of Physics: Condensed Matter. 20 (2008) 145212. (arXiv:0907.1837)

[28] M. Edelman, V.E. Tarasov, Physics Letters A 374 (2009) 279-285. (arXiv:0909.5412) 